Eur. J. Math. Anal. 1 (2021) 68-85

doi: 10.28924/ada/ma.1.68

\title{
Unified Convergence Analysis of Two-Step Iterative Methods for Solving Equations
}

\author{
loannis K. Argyros \\ Department of Mathematical Sciences, Cameron University, Lawton, OK 73505, USA \\ Correspondence: iargyros@cameron.edu
}

\begin{abstract}
AвSTRACT. In this paper we consider unified convergence analysis of two-step iterative methods for solving equations in the Banach space setting. The convergence order four was shown using Taylor expansions requiring the existence of the fifth derivative not on this method. But these hypotheses limit the utilization of it to functions which are at least five times differentiable although the method may converge. As far as we know no semi-local convergence has been given in this setting. Our goal is to extend the applicability of this method in both the local and semi-local convergence case and in the more general setting of Banach space valued operators. Moreover, we use our idea of recurrent functions and conditions only on the first derivative and divided differences which appear on the method. This idea can be used to extend other high convergence multipoint and multistep methods. Numerical experiments testing the convergence criteria complement this study.
\end{abstract}

\section{INTRODUCTION}

We consider the problem of approximating a solution $x^{*}$ of equation

$$
F(x)=0
$$

where $F: \Omega \subset B \longrightarrow B_{1}$ is a continuous operator acting between Banach spaces $B$ and $B_{1}$ with $\Omega \neq \emptyset$. Since a closed form solution is not possible in general, iterative methods are used for solving (1.1). Many iterative methods are studied for approximating $x^{*}$. In this paper, we consider the iterative methods, defined for $n=0,1,2, \ldots$, by

$$
\begin{aligned}
y_{n} & =x_{n}-F^{\prime}\left(x_{n}\right)^{-1} F\left(x_{n}\right) \\
x_{n+1} & =y_{n}-A_{n} F^{\prime}\left(x_{n}\right)^{-1} F\left(y_{n}\right),
\end{aligned}
$$

$A_{n}=A\left(x_{n}, y_{n}\right), A: \Omega \times \Omega \longrightarrow L\left(B, B_{1}\right)$, where $A^{-1} \in L\left(B_{1}, B\right)$. Many methods are special cases of (1.2). For example:

Received: 31 Aug 2021.

Key words and phrases. iterative methods; Banach space; convergence criterion; continuous functions. 
Traub [35]

$$
\begin{aligned}
y_{n} & =x_{n}-F^{\prime}\left(x_{n}\right)^{-1} F\left(x_{n}\right) \\
x_{n+1} & =y_{n}-F^{\prime}\left(x_{n}\right)^{-1} F\left(y_{n}\right),
\end{aligned}
$$

Newton [6]

$$
\begin{aligned}
y_{n} & =x_{n}-F^{\prime}\left(x_{n}\right)^{-1} F\left(x_{n}\right) \\
x_{n+1} & =y_{n}-F^{\prime}\left(y_{n}\right)^{-1} F\left(y_{n}\right),
\end{aligned}
$$

Ostrowski [25]

$$
\begin{aligned}
y_{n} & =x_{n}-F^{\prime}\left(x_{n}\right)^{-1} F\left(x_{n}\right) \\
x_{n+1} & =y_{n}-\left(2\left[x_{n}, y_{n} ; F\right]-F^{\prime}\left(x_{n}\right)\right)^{-1} F\left(y_{n}\right),
\end{aligned}
$$

Kung-Traub [35-37]

$$
\begin{aligned}
y_{n} & =x_{n}-F^{\prime}\left(x_{n}\right)^{-1} F\left(x_{n}\right) \\
x_{n+1} & =y_{n}-\left[x_{n}, y_{n} ; F\right]^{-1} F^{\prime}\left(x_{n}\right)\left[x_{n}, y_{n} ; F\right]^{-1} F\left(y_{n}\right),
\end{aligned}
$$

Ostrowski-type [25]

$$
\begin{aligned}
y_{n} & =x_{n}-F^{\prime}\left(x_{n}\right)^{-1} F\left(x_{n}\right) \\
x_{n+1} & =y_{n}-\left(2\left[x_{n}, y_{n} ; F\right]^{-1}-F^{\prime}\left(x_{n}\right)^{-1}\right) F\left(y_{n}\right),
\end{aligned}
$$

Sharma type [32]

$$
\begin{aligned}
y_{n} & =x_{n}-F^{\prime}\left(x_{n}\right)^{-1} F\left(x_{n}\right) \\
x_{n+1} & =y_{n}-P\left(x_{n}, y_{n}\right) F^{\prime}\left(x_{n}\right)^{-1} F\left(y_{n}\right) .
\end{aligned}
$$

To obtain all these special cases choose, $A_{n}=1, A_{n}=F^{\prime}\left(y_{n}\right)^{-1} F^{\prime}\left(x_{n}\right), A_{n}=\left(2\left[x_{n}, y_{n} ; F\right]-\right.$ $\left.F^{\prime}\left(x_{n}\right)\right) F^{\prime}\left(x_{n}\right), A_{n}=\left[x_{n}, y_{n} ; F\right]^{-1} F^{\prime}\left(x_{n}\right)\left[x_{n}, y_{n} ; F\right]^{-1} F^{\prime}\left(x_{n}\right), A_{n}=\left(2\left[x_{n}, y_{n} ; F\right]^{-1}-F^{\prime}\left(x_{n}\right)^{-1}\right) F^{\prime}\left(x_{n}\right)$, $A_{n}=P\left(x_{n}, y_{n}\right)$, respectively, where $[., ; F]: \Omega \times \Omega \longrightarrow L\left(B, B_{1}\right)$ is a divided difference of order one and $P: \Omega \times \Omega \longrightarrow L\left(B, B_{1}\right)$ is weight operator [32] (see also $[15,28,40]$ and reference therein). These special methods were shown to be of order four using Taylor expansion and assumptions on the fifth order derivative of $F$, which is not on these methods. So, the assumptions on the fifth derivative reduce the applicability of these methods [1-41].

For example: Let $B=B_{1}=\mathbb{R}, \Omega=[-0.5,1.5]$. Define $\lambda$ on $\Omega$ by

$$
\lambda(t)=\left\{\begin{array}{cl}
t^{3} \log t^{2}+t^{5}-t^{4} & \text { if } t \neq 0 \\
0 & \text { if } t=0
\end{array}\right.
$$

Then, we get $t^{*}=1$, and

$$
\lambda^{\prime \prime \prime}(t)=6 \log t^{2}+60 t^{2}-24 t+22
$$


Obviously $\lambda^{\prime \prime \prime}(t)$ is not bounded on $\Omega$. So, the convergence of method (1.2) is not guaranteed by the previous analyses in [1-41].

In this paper we introduce a majorant sequence and use our idea of recurrent functions to extend the applicability of method (1.2). Our analysis includes error bounds and results on uniqueness of $x^{*}$ based on computable Lipschitz constants not given before in [1-41] and in other similar studies using Taylor series. Our idea is very general. So, it applies on other methods too.

The rest of the paper is set up as follows: In Section 2 we present results on majorizing sequences. Sections 3,4 contain the semi-local and local convergence, respectively, where in Section 4 the numerical experiments are presented. Concluding remarks are given in the last Section 5.

\section{Results on majorizInG SEQUences}

We recall the definition followed by convergence results.

Definition 2.1. Let $\left\{\bar{w}_{n}\right\}$ be a sequence in a Banach space. Then, a nondecreasing scalar sequence $\left\{w_{n}\right\}$ is called majorizing for $\left\{\bar{w}_{n}\right\}$ if

$$
\left\|\bar{w}_{n+1}-\bar{w}_{n}\right\| \leq w_{n+1}-w_{n} \text { for each } n=0,1,2, \ldots
$$

Sequence $\left\{w_{n}\right\}$ is used instead to study the convergence of $\left\{\bar{w}_{n}\right\}[23-25]$. Set $M=[0, \infty)$.

Let $\eta>0, P_{0}: M \longrightarrow \mathbb{R}, P: M \longrightarrow \mathbb{R}, a: M \times M \times M \longrightarrow \mathbb{R}, \bar{a}: M \times M \times M \longrightarrow \mathbb{R}$ and $b: M \times M \times M \times M \longrightarrow \mathbb{R}$ be continuous and nondecreasing functions. Set $a_{n}=a(n)$ and $\xi_{n}=b(n)$. Define scalar sequences $\left\{s_{n}\right\},\left\{t_{n}\right\}$ for each $n=0,1,2, \ldots$ by $t_{0}=0, s_{0}=\eta$,

$$
\begin{aligned}
& t_{n+1}=s_{n}+\bar{\alpha}_{n}\left(s_{n}-t_{n}\right) \\
& s_{n+1}=t_{n+1}+\beta_{n}\left(t_{n+1}-s_{n}\right),
\end{aligned}
$$

where $\bar{\alpha}_{n}=\bar{a}_{n} \int_{0}^{1} \bar{P}\left((1-\theta)\left(s_{n}-t_{n}\right)\right) d \theta$ and

$$
\begin{gathered}
\beta_{n}=\frac{\xi_{n}}{1-P_{0}\left(t_{n+1}\right)}, \bar{a}_{n}=\left\{\begin{array}{l}
\bar{a}, \quad \text { if } n=0 \\
a, \quad \text { if } n=1,2, \ldots,
\end{array}\right. \\
\bar{P}=\left\{\begin{array}{c}
P_{0}, \quad \text { if } n=0 \\
P, \quad \text { if } n=1,2, \ldots
\end{array}\right.
\end{gathered}
$$

Next, we present results on the convergence of sequence $\left\{s_{n}\right\},\left\{t_{n}\right\}$.

LEMMA 2.2. Suppose that there exists $\mu>0$ such that for each $n=0,1,2, \ldots$,

$$
t_{n} \leq \mu
$$

and

$$
P_{0}(\mu)<1
$$


Then, sequences $\left\{s_{n}\right\},\left\{t_{n}\right\}$ converge to their unique least upper bound $t^{*} \in[\eta, \mu]$ and

$$
t_{n} \leq s_{n} \leq t_{n+1}
$$

Proof. It follows from (2.2)-(2.4) that these sequences are nondecreasing, bounded from above by $\mu$, and as such they converge to $t^{*}$.

LEMMA 2.3. If function $P_{0}$ is increasing then conditions (2.3) and (2.4) can be replaced by

$$
t_{n} \leq P_{0}^{-1}(1)
$$

Proof. Set $\mu=P_{0}^{-1}(1)$ in Lemma 2.2.

REMARK 2.4. Conditions (2.3)-(2.5) are very general and can be verified only in special cases. That is why we present stronger conditions that are easier to verify.

Define functions $f$ and $g$ on the interval $[0,1)$ by

$$
f(t)=a\left(\frac{\eta}{1-t}, \frac{\eta}{1-t}, t^{2} \eta\right) \int_{0}^{1} P\left((1-\theta) t^{2} \eta\right) d \theta-t
$$

and

$$
g(t)=b\left(\frac{\eta}{1-t}, \frac{\eta}{1-t}, t^{2} \eta, t^{3} \eta\right)+t P_{0}\left(\frac{\eta}{1-t}\right)-t
$$

Suppose that these functions have minimal zeros $\lambda_{f}$ and $\lambda_{g}$ in $(0,1)$, respectively. Set $\lambda=$ $\min \left\{\lambda_{f}, \lambda_{g}\right\}$ and $\lambda_{0}=\max \left\{\alpha_{0}, \beta_{0}\right\}$. Then, we can show the third result on majorizing sequence for method (1.2).

LEMMA 2.5. Suppose that

$$
\mu_{0} \leq \lambda_{0} \leq \lambda
$$

Then, sequences $\left\{s_{n}\right\},\left\{t_{n}\right\}$ are nondecreasing, bounded from above by $t^{* *}=\frac{\eta}{1-\lambda}$, and converge to $t^{*} \in\left[0, t^{* *}\right]$. Moreover, the following estimates hold for each $n=1,2, \ldots$

$$
\begin{gathered}
0 \leq s_{n}-t_{n} \leq \lambda\left(t_{n}-s_{n-1}\right) \leq \lambda^{2 n} \eta \\
0 \leq t_{n+1}-s_{n} \leq \lambda\left(s_{n}-t_{n}\right) \leq \lambda^{2 n+1} \eta, \\
0 \leq s_{n} \leq \frac{1-\lambda^{2 n+1}}{1-\lambda} \eta
\end{gathered}
$$

and

$$
0 \leq t_{n+1} \leq \frac{1-\lambda^{2 n+1}}{1-\lambda} \eta
$$


Proof. Estimates (2.7)-(2.10) hold if

$$
\begin{aligned}
& 0 \leq \alpha_{m} \leq \lambda, \\
& 0 \leq \beta_{m} \leq \lambda,
\end{aligned}
$$

and

$$
t_{m} \leq s_{m} \leq t_{m+1}
$$

are true for $m=0,1,2, \ldots$ These estimates hold for $m=0$ by (2.6). We suppose that (2.11)(2.13) are true for $m=1,2, \ldots n$. By induction hypotheses, (2.7) and (2.8), we have

$$
\begin{aligned}
s_{m} & \leq t_{m}+\lambda^{2 m} \eta \leq s_{m-1}+\lambda^{2 m-1} \eta+\lambda^{2 m} \eta \\
& \leq \eta+\lambda \eta+\ldots+\lambda^{2 m} \eta \\
& =\frac{1-\lambda^{2 m+1}}{1-\lambda} \eta<\frac{\eta}{1-\lambda}=t^{* *}
\end{aligned}
$$

and

$$
\begin{aligned}
t_{m+1} & \leq s_{m}+\lambda^{2 m+1} \eta \leq t_{m}+\lambda^{2 m} \eta+\lambda^{2 m+1} \eta \\
& \leq \eta+\lambda \eta+\ldots+\lambda^{2 m+1} \eta \\
& =\frac{1-\lambda^{2 m+2}}{1-\lambda} \eta<\frac{\eta}{1-\lambda}=t^{* *} .
\end{aligned}
$$

Therefore, by (2.13) and the induction hypotheses, we see that sequences $\left\{s_{m}\right\}$ and $\left\{t_{m}\right\}$ are nondecreasing. Then, (2.11) shall be true if

$$
a\left(t_{m}, s_{m}, s_{m}-t_{m}\right) \int_{0}^{1} \psi\left((1-\theta)\left(s_{m}-t_{m}\right)\right) d \theta \leq \lambda
$$

or

$$
a\left(\frac{1-\lambda^{2 m}}{1-\lambda} \eta, \frac{1-\lambda^{2 m+1}}{1-\lambda} \eta, \lambda^{2 m} \eta\right) \int_{0}^{1} P\left((1-\theta) \lambda^{2 m} \eta\right) d \theta \leq \lambda
$$

or

$$
a\left(\frac{\eta}{1-\lambda}, \frac{\eta}{1-\lambda}, \lambda^{2} \eta\right) \int_{0}^{1} \psi\left((1-\theta) \lambda^{2} \eta\right) d \theta \leq \lambda
$$

or

$$
f(\lambda) \leq 0,
$$

which is true by the definition of $\lambda_{f}$ and $\lambda$. Similarly, (2.12) shall be true if

$$
\begin{aligned}
& b\left(\frac{1-\lambda^{2 m}}{1-\lambda} \eta, \frac{1-\lambda^{2 m}}{1-\lambda} \eta, \lambda^{2 m} \eta, \lambda^{2 m+1} \eta\right) \\
& +\lambda P_{0}\left(\frac{1-\lambda^{2 m+2}}{1-\lambda} \eta\right) \leq \lambda,
\end{aligned}
$$

or

$$
b\left(\frac{\eta}{1-\lambda}, \frac{\eta}{1-\lambda}, \lambda^{2} \eta, \lambda^{3} \eta\right)+\lambda P_{0}\left(\frac{\eta}{1-\lambda}\right) \leq \lambda
$$


or

$$
g(\lambda) \leq 0
$$

which is also true by the definition of $\lambda_{g}$ and $\lambda$. Hence, we conclude (2.13) holds and $\lim _{m} \longrightarrow \infty S_{m}=$ $\lim _{m \longrightarrow \infty} t_{m}=t^{*}$.

\section{Semi-Local conVERGENCE}

Let $U\left(x_{0}, r\right)=\left\{x \in B:\left\|x-x_{0}\right\|<r, r>0\right\}$ and $U\left[x_{0}, r\right]=\left\{x \in B:\left\|x-x_{0}\right\| \leq r, r>0\right\}$. We use some parameters and functions. Consider $M=[0, \infty)$. Suppose that there exists function $P_{0}: M \longrightarrow M$ which is continuous and nondecreasing such that functions $P_{0}(t)-1=0$ has a minimal zero $s \in(0, \infty)$. Set $M_{0}=[0, s)$. Suppose function $P_{0}: M_{0} \longrightarrow M$ is continuous and nondecreasing. The following conditions $(\mathrm{C})$ are needed:

(C1) There exists $x_{0} \in \Omega$ and $\eta>0$ such that $F^{\prime}\left(x_{0}\right)^{-1} \in L\left(B_{1}, B\right)$ and

$$
\left\|F^{\prime}\left(x_{0}\right)^{-1} F\left(x_{0}\right)\right\| \leq \eta
$$

(C2) For each $x \in \Omega$

$$
\left\|F^{\prime}\left(x_{0}\right)^{-1}\left(F^{\prime}(u)-F^{\prime}\left(x_{0}\right)\right)\right\| \leq P_{0}\left(\left\|u-x_{0}\right\|\right) .
$$

Set $S_{0}=U\left(x_{0}, s\right) \cap \Omega$.

(C3) For each $x, y \in S_{0}$ the following hold

$$
\left\|F^{\prime}\left(x_{0}\right)^{-1}\left(F^{\prime}(y)-F^{\prime}(x)\right)\right\| \leq P(\|y-x\|)
$$

(C4) For each $n=0,1,2, \ldots$

$$
\begin{gathered}
\left\|A_{n} F^{\prime}\left(x_{n}\right)^{-1} F^{\prime}\left(x_{0}\right)\right\| \leq a_{n} \\
F^{\prime}\left(x_{0}\right)^{-1}\left([y, x ; F]-F^{\prime}(x)\right)\left\|\leq L_{2}\right\| y-x \|
\end{gathered}
$$

and

$$
\left\|F^{\prime}\left(x_{0}\right)^{-1} H_{n}\right\| \leq \xi_{n}
$$

where

$$
H_{n}=F^{\prime}\left(x_{0}\right)^{-1} \int_{0}^{1}\left(F^{\prime}\left(y_{n}+\theta\left(x_{n+1}-y_{n}\right)\right)-F^{\prime}\left(x_{n}\right) A_{n}^{-1}\right) d \theta .
$$

(C5) Conditions of Lemma 2.2 or Lemma 2.3 or Lemma 2.5 hold. and

(C6) $U\left[x_{0}, t^{*}\right] \subset \Omega$.

Then, we can show the semi-local convergence of method (1.2) using the conditions (C) and the preceding notation. 
THEOREM 3.1. Under the conditions (C), sequences $\left\{y_{n}\right\},\left\{x_{n}\right\}$ generated by method (1.2) are well defined in $U\left[x_{0}, t^{*}\right]$, remain in $U\left[x_{0}, t^{*}\right]$ for each $n=0,1,2, \ldots$ and converge to a solution $x^{*} \in U\left[x_{0}, t^{*}\right]$ of equation $F(x)=0$. Moreover, the following error estimates hold for each $n=$ $0,1,2, \ldots$

$$
\left\|x^{*}-x_{n}\right\| \leq t^{*}-t_{n}
$$

Proof. We shall show items

$$
\begin{aligned}
& \left(P_{m}\right)\left\|y_{m}-x_{m}\right\| \leq s_{m}-t_{m} \\
& \left(Q_{m}\right)\left\|x_{m+1}-y_{m}\right\| \leq t_{m+1}-s_{m}
\end{aligned}
$$

using mathematical induction on integer $m$. By the first substep of method (1.2) for $n=0$ and (C1), we have

$$
\left\|y_{0}-x_{0}\right\|=\left\|F^{\prime}\left(x_{0}\right)^{-1} F\left(x_{0}\right)\right\| \leq \eta=s_{0}-t_{0}=s_{0} \leq t^{*},
$$

so $y_{0} \in U\left[x_{0}, t^{*}\right]$ and $\left(P_{0}\right)$ holds. We can write by the first sustep of method (1.2) that

$$
F\left(y_{0}\right)=F\left(y_{0}\right)-F\left(x_{0}\right)-F^{\prime}\left(x_{0}\right)\left(y_{0}-x_{0}\right)=\int_{0}^{1}\left(F^{\prime}\left(x_{0}+\theta\left(y_{0}-x_{0}\right)\right)-F^{\prime}\left(x_{0}\right)\right)\left(y_{0}-x_{0}\right) d \theta,
$$

leading by $(\mathrm{C} 2)$ and $\left(P_{0}\right)$ to

$$
\begin{aligned}
\left\|F^{\prime}\left(x_{0}\right)^{-1} F\left(y_{0}\right)\right\| & \leq \int_{0}^{1} P_{0}\left(\theta\left\|y_{0}-x_{0}\right\|\right) d \theta\left\|y_{0}-x_{0}\right\| \\
& \leq \int_{0}^{1} \bar{P}\left(\theta\left(s_{0}-t_{0}\right)\right) d \theta\left(s_{0}-t_{0}\right) .
\end{aligned}
$$

Let $z \in U\left(x_{0}, t^{*}\right)$. In view of $(C 2)$, we get

$$
\begin{aligned}
\left\|F^{\prime}\left(x_{0}\right)^{-1}\left(F^{\prime}(z)-F^{\prime}\left(x_{0}\right)\right)\right\| & \leq P_{0}\left(\left\|z-x_{0}\right\|\right) \\
& \leq P_{0}\left(t^{*}\right)<1
\end{aligned}
$$

so

$$
\left\|F^{\prime}(z)^{-1} F^{\prime}\left(x_{0}\right)\right\| \leq \frac{1}{1-P_{0}\left(\left\|z-x_{0}\right\|\right)}
$$

holds by a lemma on invertible linear operators due to Banach [24] and (3.2). Therefore, iterate $x_{1}$ is well defined and we can write in turn by (C3) and (3.3) (for $z=x_{0}, y_{0}$ )

$$
\begin{aligned}
\left\|x_{1}-y_{0}\right\| & =\left\|A_{0} F^{\prime}\left(x_{0}\right)^{-1} F\left(y_{0}\right)\right\| \\
& \leq\left\|A_{0} F^{\prime}\left(x_{0}\right)^{-1} F^{\prime}\left(x_{0}\right)\right\|\left\|\int_{0}^{1} F^{\prime}\left(x_{0}\right)^{-1}\left(F^{\prime}\left(x_{0}+\theta\left(y_{0}-x_{0}\right)\right)-F^{\prime}\left(x_{0}\right)\right) d \theta\left(y_{0}-x_{0}\right)\right\| \\
& \leq \frac{a_{0} \int_{0}^{1} \bar{P}\left((1-\theta)\left\|y_{0}-x_{0}\right\|\right) d \theta\left\|y_{0}-x_{0}\right\|}{1-P_{0}\left(\left\|x_{0}-x_{0}\right\|\right)} \\
& \leq \frac{a_{0} \int_{0}^{1} \bar{P}\left((1-\theta)\left(s_{0}-t_{0}\right)\right) d \theta}{1-P_{0}(0)}\left(s_{0}-t_{0}\right)=t_{1}-s_{0}
\end{aligned}
$$


showing $\left(Q_{0}\right)$. Then, we have

$$
\left\|x_{1}-x_{0}\right\| \leq\left\|x_{0}-y_{0}\right\|+\left\|y_{0}-x_{0}\right\| \leq t_{1}-s_{0}+s_{0}-t_{0}=t_{1} \leq t^{*}
$$

so $x_{1} \in U\left[x_{0}, t^{*}\right]$. Moreover, we can write

$$
\begin{aligned}
F\left(x_{1}\right) & =F\left(x_{1}\right)-F\left(y_{0}\right)+F\left(y_{0}\right) \\
& =F\left(x_{1}\right)-F\left(y_{0}\right)-F^{\prime}\left(x_{0}\right) A_{0}^{-1}\left(x_{1}-y_{0}\right) \\
& =\int_{0}^{1}\left(F^{\prime}\left(y_{0}+\theta\left(x_{1}-x_{0}\right)\right)-F^{\prime}\left(x_{0}\right) A_{0}^{-1}\right) d \theta\left(x_{1}-y_{0}\right) \\
& =H_{0}\left(x_{1}-y_{0}\right),
\end{aligned}
$$

since by the second substep of method (1.2), we have $F\left(y_{0}\right)=-F^{\prime}\left(x_{0}\right) A_{0}^{-1}\left(x_{1}-y_{0}\right)$. By (C3), (3.4) and (3.5), we obtain

$$
\begin{aligned}
\left\|F^{\prime}\left(x_{0}\right)^{-1} F\left(x_{1}\right)\right\| & \leq\left\|F^{\prime}\left(x_{0}\right)^{-1} H_{0}\right\|\left\|x_{1}-y_{0}\right\| \\
& \leq \xi_{0}\left(t_{1}-s_{0}\right)
\end{aligned}
$$

so

$$
\begin{aligned}
\left\|y_{1}-x_{1}\right\| & \leq\left\|F^{\prime}\left(x_{1}\right)^{-1} F^{\prime}\left(x_{0}\right)\right\|\left\|F^{\prime}\left(x_{0}\right)^{-1} F\left(x_{1}\right)\right\| \\
& \leq \frac{\xi_{0}\left(t_{1}-s_{0}\right)}{1-P_{0}\left(t_{1}\right)}=s_{1}-t_{1}
\end{aligned}
$$

showing $\left(P_{1}\right)$ for $m=1$. Suppose $\left(P_{m}\right),\left(Q_{m}\right)$ hold $y_{m}$ and $x_{m+1} \in U\left[x_{0}, t^{*}\right]$. Then, by repeating these computations with $x_{m}, y_{m}, x_{m+1}$ replacing $x_{0}, y_{0}, x_{1}$, respectively, we complete the induction. Moreover, sequence $\left\{x_{m}\right\}$ is complete in a Banach space, so it converges to some $x^{*} \in U\left[x_{0}, t^{*}\right]$.

Finally, by letting $m \longrightarrow \infty$ in the estimation

$$
\left\|F^{\prime}\left(x_{0}\right)^{-1} F\left(x_{m+1}\right)\right\| \leq \xi_{m}\left(t_{m+1}-s_{m}\right)
$$

and using the continuity of $F$, we conclude $F\left(x^{*}\right)=0$.

Next, we present a result for uniqueness of the solution $x^{*}$.

\section{PROPOSITION 3.2. Suppose}

(a) $x^{*}$ is a solution of $F(x)=0$

(b) there exists $\tilde{s} \geq t^{*}$ such that

$$
\int_{0}^{1} P_{0}\left((1-\theta) \tilde{s}+\theta t^{*}\right) d \theta<1 .
$$

Set $S_{1}=U\left[x_{0}, \tilde{s}\right] \cap \Omega$. Then, the only solution of equation $F(x)=0$ in the region $S_{1}$ is $x^{*}$. 
Proof. Set $T=\int_{0}^{1} F^{\prime}\left(\tilde{x}+\theta\left(x^{*}-\tilde{x}\right)\right) d \theta$ for some $\tilde{x} \in S_{1}$ with $F(\tilde{x})=0$. Using (C2) and (3.9), we get

$$
\begin{aligned}
\left\|F^{\prime}\left(x_{0}\right)^{-1}\left(T-F^{\prime}\left(x_{0}\right)\right)\right\| & \leq \int_{0}^{1} P_{0}\left(\left\|\tilde{x}+\theta\left(x^{*}-\tilde{x}\right)-x_{0}\right\| d \theta\right. \\
& \leq \int_{0}^{1} P_{0}\left((1-\theta)\left\|\tilde{x}-x_{0}\right\|+\theta\left\|x^{*}-x_{0}\right\|\right) d \theta \\
& \leq \int_{0}^{1} P_{0}\left((1-\theta) \tilde{s}+\theta t^{*}\right) d \theta<1,
\end{aligned}
$$

leading to $\tilde{x}=x^{*}$, where we used the identity $T\left(x^{*}-\tilde{x}\right)=F\left(x^{*}\right)-F(\tilde{x})=0-0=0$ and the invertability of $T$.

REMARK 3.3. Let us specialize operators $A_{n}$ to see how sequences $\left\{s_{n}\right\},\left\{t_{n}\right\},\left\{a_{n}\right\},\left\{\xi_{n}\right\},\left\{\alpha_{n}\right\}$ and $\left\{\beta_{n}\right\}$ are defined. Choose the case of Newton's method (1.4). Then, we have

$$
\begin{aligned}
\left\|A_{n} F^{\prime}\left(x_{n}\right)^{-1} F^{\prime}\left(x_{0}\right)\right\| & =\left\|F^{\prime}\left(y_{n}\right)^{-1} F^{\prime}\left(x_{0}\right)\right\| \\
& \leq \frac{1}{1-P_{0}\left(\left\|y_{n}-x_{0}\right\|\right)}
\end{aligned}
$$

and

$$
\begin{aligned}
\left\|F^{\prime}\left(x_{0}\right)^{-1} H_{n}\right\| & =\left\|\int_{0}^{1} F^{\prime}\left(x_{0}\right)^{-1}\left(F^{\prime}\left(y_{n}+\theta\left(x_{n+1}-y_{n}\right)\right)-F^{\prime}\left(x_{n}\right) A_{n}^{-1}\right) d \theta\right\| \\
& =\left\|\int_{0}^{1} F^{\prime}\left(x_{0}\right)^{-1}\left(F^{\prime}\left(y_{n}+\theta\left(x_{n+1}-y_{n}\right)\right)-F^{\prime}\left(y_{n}\right)\right) d \theta\right\| \\
& \leq \int_{0}^{1} \bar{P}\left(\theta\left\|x_{n+1}-y_{n}\right\|\right) d \theta \\
& \leq \int_{0}^{1} \bar{P}\left(\theta\left(t_{n+1}-s_{n}\right)\right) d \theta
\end{aligned}
$$

so we can choose

$$
a_{n}=\frac{1}{1-P_{0}\left(s_{n}\right)}
$$

and

$$
\xi_{n}=\int_{0}^{1} \bar{P}\left(\theta\left(t_{n+1}-s_{n}\right)\right) d \theta .
$$

In this case we can show another result on majorizing sequences which is weaker than Lemma 2.5 for the interesting case $P_{0}(t)=L_{0} t$ and $P(t)=L t$. We get in this special case that

$$
\alpha_{n}=\frac{L\left(s_{n}-t_{n}\right)}{2\left(1-L_{0} s_{n}\right)}
$$

and

$$
\beta_{n}=\frac{L\left(t_{n+1}-s_{n}\right)}{2\left(1-L_{0} t_{n+1}\right)}
$$


Define sequences of function $\left\{f_{n}^{(1)}\right\},\left\{f_{n}^{(2)}\right\}$ on the interval $[0,1)$ by

$$
\begin{aligned}
& f_{n}^{(1)}(t)=\frac{L}{2} t^{2 n-1} \eta+L_{0}\left(1+t+\ldots+t^{2 n}\right) \eta-1, \\
& f_{n}^{(2)}(t)=\frac{L}{2} t^{2 n} \eta+L_{0}\left(1+t+\ldots+t^{2 n+1}\right) \eta-1,
\end{aligned}
$$

and polynomial $\varphi$ by

$$
\varphi(t)=L_{0} t^{3}+\left(L_{0}+\frac{L}{2}\right) t^{2}-\frac{L}{2} .
$$

Notice that $\varphi(0)=-\frac{L}{2}$ and $\varphi(1)=2 L_{0}$. Denote by $\rho$ the smallest zero of polynomial $\varphi$ in $(0,1)$ assured to exist by the intermediate value theorem.

LEMMA 3.4. Suppose that

$$
\lambda_{0} \leq \rho<1-L_{0} \eta .
$$

Then, the conclusions of Lemma 2.5 hold for sequences $\left\{s_{n}\right\},\left\{t_{n}\right\}$ with $\rho$ replacing $\lambda$.

Proof. We must show this time

$$
\begin{gathered}
0 \leq \frac{L\left(s_{m}-t_{m}\right)}{2\left(1-L_{0} s_{m}\right)} \leq \rho, \\
0 \leq \frac{L\left(t_{m+1}-s_{m}\right)}{2\left(1-L_{0} t_{m+1}\right)} \leq \rho
\end{gathered}
$$

and

$$
t_{m} \leq s_{m} \leq t_{m+1}
$$

These estimates hold for $m=0$ by (3.14) and the definition of these sequences. Then, as in Lemma 2.5 we can show instead for (3.15) that

$$
\frac{L}{2} \rho^{2 m} \eta+\rho L_{0}\left(1+\rho+\ldots+\rho^{2 m}\right) \eta-1 \leq 0 .
$$

This estimate motivates us to define recurrent functions $f_{m}^{(1)}$ by

$$
f_{m}^{(1)}(t)=\frac{L}{2} t^{2 m-1} \eta+L_{0}\left(1+t+\ldots+t^{2 m}\right) \eta-1 .
$$

We shall find a relationship between recurrent functions $f_{m+1}^{(1)}$ and $f_{m}^{(1)}$. By definition (3.9), we have in turn that

$$
\begin{aligned}
f_{m+1}^{(1)}(t)= & \frac{L}{2} t^{2 m+1} \eta+L_{0}\left(1+t+\ldots+t^{2 m+2}\right) \eta-1 \\
& -\frac{L}{2} t^{2 m-1} \eta-L_{0}\left(1+t+\ldots+t^{2 m}\right) \eta+1+f_{m}^{(1)}(t) \\
= & f_{m}^{(1)}(t)+\left(\frac{L}{2} t^{2}-\frac{L}{2}+L_{0}\left(t^{2}+t^{3}\right)\right) t^{2 m-1} \eta \\
= & f_{m}^{(1)}(t)+p(t) t^{2 m-1} \eta .
\end{aligned}
$$

In particular, we have

$$
f_{m+1}(\rho)=f_{m}(\rho)
$$


so evidently (3.8) holds if

$$
f_{m}^{(1)}(\rho) \leq 0
$$

Define $f_{\infty}^{(1)}(t)=\lim _{m \longrightarrow \infty} f_{m}^{(1)}(t)$. Then, we have

$$
f_{\infty}(t)=\frac{L_{0} \eta}{1-t}-1
$$

Then, (3.22) holds if

$$
f_{\infty}(\rho) \leq 0,
$$

which is true by (3.14). Similarly, (3.16) holds if

$$
\frac{L}{2} \rho^{2 m+1} \eta+\rho L_{0}\left(1+\rho+\ldots+\rho^{2 m+1}\right) \eta-\rho \leq 0
$$

or

$$
f_{m}^{(2)}(\rho) \leq 0
$$

As in (3.20), we get in turn that

$$
\begin{aligned}
f_{m+1}^{(2)}(t)= & \frac{L}{2} t^{2 m+2} \eta+L_{0}\left(1+t+\ldots+t^{2 m+3}\right) \eta-1 \\
& -\frac{L}{2} t^{2 m} \eta-L_{0}\left(1+t+\ldots+t^{2 m+1}\right) \eta+1+f_{m}^{(2)}(t) \\
= & f_{m}^{(2)}(t)+\varphi(t) t^{2 m} \eta .
\end{aligned}
$$

Define $f_{\infty}^{(2)}(t)=\lim _{m \longrightarrow \infty}^{(2)}(t)$. Then, we get again

$$
f_{\infty}^{(2)}(t)=f_{\infty}^{(1)}(t)
$$

so

$$
f_{\infty}^{(2)}(\rho) \leq 0
$$

can be shown instead of (3.26). But this is true by (3.14). The induction for items (3.15)-(3.17) is completed. The rest of the proof follows as in Lemma 2.2.

\section{Local Convergence}

We shall introduce real parameters and functions to be used in the convergence analysis. Set $M=[0, \infty)$.

Suppose function

(i) $\psi_{0}(t)-1=0$ has a smallest zero $R_{0} \in M-\{0\}$, where function $\psi_{0}: M \longrightarrow M$ is continuous and nondecreasing. Set $M_{0}=\left[0, R_{0}\right)$.

(ii) $\psi_{1}(t)-1=0$, has a smallest zero $R_{1} \in M_{0}-\{0\}$, where function $\psi: M_{0} \longrightarrow M$ is continuous and nondecreasing and $\psi_{1}: M_{0} \longrightarrow M$ is defined by

$$
\psi_{1}(t)=\frac{\int_{0}^{1} \psi((1-\theta) t) d \theta}{1-\psi_{0}(t)}
$$


(iii) $\psi_{0}\left(\psi_{1}(t) t\right)-1$ has a smallest zero $\bar{R}_{1} \in M_{0}-\{0\}$. Ser $\bar{R}_{2}=\min \left\{R_{0}, \bar{R}_{1}\right\}$ and $M_{1}=\left[0, \bar{R}_{2}\right)$. (iv) $\psi_{2}(t)-1=0$ has a smallest zero $R_{2} \in M_{1}-\{0\}$, where

$$
\psi_{2}(t)=\left[\psi_{1}\left(\psi_{1}(t) t\right)+\frac{\left(\psi_{0}(t)+h\left(t, \psi_{1}(t) t\right)\right) \int_{0}^{1} \omega\left(\theta \psi_{1}(t) t\right) d \theta}{\left(1-\psi_{0}(t)\right)\left(1-\psi_{0}\left(\psi_{1}(t) t\right)\right)}\right] \psi_{1}(t),
$$

where $\omega: M_{1} \longrightarrow M$ and $h: M \times M_{1} \longrightarrow M$ are continuous and nondecreasing. We shall show that

$$
R=\min \left\{R_{1}, R_{2}\right\}
$$

is a convergence radius for method (1.2). Set $M_{2}=[0, R)$. These definitions, imply that for each $t \in M_{2}$

$$
\begin{gathered}
0 \leq \psi_{0}(t)<1, \\
0 \leq \psi_{0}\left(\psi_{1}(t) t\right)<1,
\end{gathered}
$$

and

$$
0 \leq \psi_{i}(t)<1, i=1,2 \text {. }
$$

The conditions $(\mathrm{H})$ shall be used provided that $x^{*}$ is a simple solution of equation $F(x)=0$.

Suppose:

(H1) For each $x \in \Omega$

$$
\left\|F^{\prime}\left(x^{*}\right)^{-1}\left(F^{\prime}(x)-F^{\prime}\left(x^{*}\right)\right)\right\| \leq \psi_{0}\left(\left\|x-x_{0}\right\|\right) .
$$

Set $\Omega_{0}=U\left(x^{*}, R_{0}\right) \cap \Omega$.

(H2) For each $x, y \in \Omega_{0}$

$$
\begin{gathered}
\left\|F^{\prime}\left(x^{*}\right)^{-1}\left(F^{\prime}(y)-F^{\prime}(x)\right)\right\| \leq \psi(\|y-x\|), \\
\left\|F^{\prime}\left(x^{*}\right)^{-1} F^{\prime}(x)\right\| \leq \omega\left(\left\|x-x^{*}\right\|\right),
\end{gathered}
$$

and

$$
\left\|F^{\prime}\left(x^{*}\right)^{-1}\left(F^{\prime}\left(x^{*}\right)-A(x, y)\right)\right\| \leq h\left(\left\|x-x^{*}\right\|,\left\|y-x^{*}\right\|\right) .
$$

(H3) $U\left[x^{*}, R\right] \subset \Omega$.

Next, we show the local convergence of method (1.2) based on the preceding notation and conditions $(\mathrm{H})$..

THEOREM 4.1. Under conditions (H) further suppose that $x_{0} \in U\left(x^{*}, R\right)-\left\{x^{*}\right\}$. Then, we conclude $\lim _{n \longrightarrow \infty} x_{n}=x^{*}$.

Proof. Let $v \in U\left(x^{*}, R\right)-\left\{x^{*}\right\}$. Using (4.1), (4.2), and (H1) we obtain in turn that

$$
\left\|F^{\prime}\left(x^{*}\right)^{-1}\left(F^{\prime}(v)-F^{\prime}\left(x^{*}\right)\right)\right\| \leq \psi_{0}\left(\left\|v-x^{*}\right\|\right) \leq \psi_{0}(R)<1,
$$

so

$$
\left\|F^{\prime}(v)^{-1} F^{\prime}\left(x^{*}\right)\right\| \leq \frac{1}{1-\psi_{0}\left(\left\|v-x^{*}\right\|\right)}
$$


In particular, iterate is well defined for $v=x_{0}$ and the first substep of method (1.2), from which we can also write

$$
\begin{aligned}
y_{0}-x^{*}= & x_{0}-x^{*}-F^{\prime}\left(x_{0}\right)^{-1} F\left(x_{0}\right) \\
= & \left(F^{\prime}\left(x_{0}\right)^{-1} F^{\prime}\left(x^{*}\right)\right) \\
& \times\left(\int_{0}^{1} F^{\prime}\left(x^{*}\right)^{-1}\left(F^{\prime}\left(x^{*}+\theta\left(x_{0}-x^{*}\right)\right)-F^{\prime}\left(x_{0}\right)\right) d \theta\left(x_{0}-x^{*}\right) .\right.
\end{aligned}
$$

By (4.1), (4.4) (for $i=1)$, (4.5) (for $\left.v=x_{0}\right),(4.6)$ and $(H 2)$, we get in turn that

$$
\begin{aligned}
\left\|y_{0}-x^{*}\right\| & \leq \frac{\int_{0}^{1} \bar{\psi}\left((1-\theta)\left\|x_{0}-x^{*}\right\|\right) d \theta\left\|x_{0}-x^{*}\right\|}{1-\psi_{0}\left(\left\|x_{0}-x^{*}\right\|\right)} \\
& \leq\left\|x_{0}-x^{*}\right\|<R,
\end{aligned}
$$

so $y_{0} \in U\left(x^{*}, R\right)$. We also have that (4.5) holds for $v=y_{0}$, and iterate $x_{1}$ is well defined from which we can write in turn that

$$
\begin{aligned}
x_{1}-x^{*}= & y_{0}-x^{*}-F^{\prime}\left(y_{0}\right)^{-1} F\left(x_{0}\right) \\
& +\left(F^{\prime}\left(y_{0}\right)^{-1}-A_{0} F^{\prime}\left(x_{0}\right)^{-1}\right) F\left(y_{0}\right) \\
= & y_{0}-x^{*}-F^{\prime}\left(y_{0}\right)^{-1} F\left(y_{0}\right)+F^{\prime}\left(y_{0}\right)^{-1}\left(F^{\prime}\left(x_{0}\right)-A_{0}\right) F^{\prime}\left(x_{0}\right)^{1} F\left(y_{0}\right) .
\end{aligned}
$$

In view of (4.1), (4.4) (for $i=2),(4.5)\left(\right.$ for $\left.v=x_{0}, y_{0}\right),(4.7),(4.8)$ and $(H 2)$, we obtain in turn

$$
\begin{aligned}
\left\|x_{1}-x^{*}\right\| \leq & {\left[\psi_{1}\left(\psi_{1}\left(\left\|x_{0}-x^{*}\right\|\right)\right)\right.} \\
& \left.+\frac{\left(\psi_{0}\left(\left\|x_{0}-x^{*}\right\|\right)+h\left(\left\|x_{0}-x^{*}\right\|,\left\|y_{0}-x^{*}\right\|\right)\right) \int_{0}^{1} \omega\left(\theta\left\|y_{0}-x^{*}\right\|\right) d \theta}{\left(1-\psi_{0}\left(\left\|y_{0}-x^{*}\right\|\right)\right)\left(1-\psi_{0}\left(\left\|x_{0}-x^{*}\right\|\right)\right)}\right]\left\|y_{0}-x^{*}\right\| \\
\leq & \psi_{2}\left(\left\|x_{0}-x^{*}\right\|\right)\left\|x_{0}-x^{*}\right\| \leq\left\|x_{0}-x^{*}\right\|<R
\end{aligned}
$$

so $x_{1} \in U\left(x^{*}, R\right)$. Simply, switch $x_{0}, y_{0}, x_{1}$ by $x_{m}, y_{m}, x_{m+1}$, respectively in the preceding calculations to get

$$
\left\|y_{m}-x^{*}\right\| \leq \psi_{1}\left(\left\|x_{m}-x^{*}\right\|\right)\left\|x_{m}-x^{*}\right\| \leq\left\|x_{m}-x^{*}\right\|<R
$$

and

$$
\left\|x_{m+1}-x^{*}\right\| \leq \psi_{2}\left(\left\|x_{m}-x^{*}\right\|\right)\left\|x_{m}-x^{*}\right\| \leq\left\|x_{m}-x^{*}\right\| .
$$

Then, by the estimation

$$
\left\|x_{m+1}-x^{*}\right\| \leq d\left\|x_{m}-x^{*}\right\|<R,
$$

where $d=\psi_{2}\left(\left\|x_{0}-x^{*}\right\|\right) \in[0,1)$, we get $\lim _{m \longrightarrow \infty} x_{m}=x^{*}$ and $x_{m+1} \in U\left(x^{*}, R\right)$.

Next, we present a uniqueness result. 
PROPOSITION 4.2. Suppose:

(i) There exists a simple solution $x^{*}$ of equation $F(x)=0$

(ii) There exists $R^{*} \geq R$ such that

$$
\int_{0}^{1} \psi_{0}\left(\theta R^{*}\right) d \theta<1
$$

Set $\Omega_{2}=\Omega \cap U\left[x^{*}, R^{*}\right]$. Then, the only solution of equation $F(x)=0$ in the region $\Omega_{2}$ is $x^{*}$.

Proof. Consider $\tilde{x} \in \Omega_{1}$ with $F(\tilde{x})=0$. Set $T=\int_{0}^{1} F^{\prime}\left(x^{*}+\theta\left(\tilde{x}-x^{*}\right)\right) d \theta$. Then, using $(\mathrm{H} 1)$ and (4.13), we get in turn that

$$
\begin{aligned}
\left\|F^{\prime}\left(x^{*}\right)^{-1}\left(T-F^{\prime}\left(x^{*}\right)\right)\right\| & \leq \int_{0}^{1} \psi_{0}\left(\theta\left\|\tilde{x}-x^{*}\right\| d \theta\right. \\
& \leq \int_{0}^{1} \psi_{0}\left(\theta R^{*}\right) d \theta<1
\end{aligned}
$$

so $\tilde{x}=x^{*}$, follows by $T^{-1} \in L\left(B_{1}, B\right)$ and $T\left(\tilde{x}-x^{*}\right)=F(\tilde{x})-F\left(x^{*}\right)=0-0=0$.

\section{Numerical Experiments}

We provide some examples in this section.

EXAMPLE 5.1. Define function

$$
q(t)=\xi_{0} t+\xi_{1}+\xi_{2} \sin \xi_{3} t, x_{0}=0
$$

where $\xi_{j}, j=0,1,2,3$ are parameters. Choose $P_{0}(t)=L_{0} t$ and $P(t)=L t$. Notice that $L_{0}$ and $L$ are the center Lipschitz and Lipschitz constants, respectively. Then, from the graph of $q(t)$ clearly for $\xi_{3}$ large and $\xi_{2}$ small, $\frac{L_{0}}{L}$ can be small (arbitrarily). Notice that $\frac{L_{0}}{L} \longrightarrow 0$.

EXAMPLE 5.2. Let $B=B_{1}=C[0,1]$ and $\Omega=U[0,1]$. It is well known that the boundary value problem [16].

$$
\begin{gathered}
\varsigma(0)=0,(1)=1, \\
\varsigma^{\prime \prime}=-\varsigma-\sigma \varsigma^{2}
\end{gathered}
$$

can be given as a Hammerstein-like nonlinear integral equation

$$
\varsigma(s)=s+\int_{0}^{1} Q(s, t)\left(s^{3}(t)+\sigma s^{2}(t)\right) d t
$$

where $\sigma$ is a parameter. Then, define $F: \Omega \longrightarrow B_{1}$ by

$$
[F(x)](s)=x(s)-s-\int_{0}^{1} Q(s, t)\left(x^{3}(t)+\sigma x^{2}(t)\right) d t
$$


Choose $\varsigma_{0}(s)=s$ and $\Omega=U\left(\varsigma_{0}, \rho_{0}\right)$. Then, clearly $U\left(\varsigma_{0}, \rho_{0}\right) \subset U\left(0, \rho_{0}+1\right)$, since $\left\|s_{0}\right\|=1$. Suppose $2 \sigma<5$. Then, conditions $(A)$ are satisfied for

$$
L_{0}=\frac{2 \sigma+3 \rho_{0}+6}{8}, L=\frac{\sigma+6 \rho_{0}+3}{4}
$$

and $\eta=\frac{1+\sigma}{5-2 \sigma}$. Notice that $L_{0}<L$.

In the last two examples we consider Traub's method (1.3). So, we take $A(x, y)=I$ and $h(s, t)=0$.

EXAMPLE 5.3. Consider the motion system

$$
G_{1}^{\prime}\left(v_{1}\right)=e^{v_{1}}, G_{2}^{\prime}(y)=(e-1) v_{2}+1, G_{3}^{\prime}\left(v_{3}\right)=1
$$

with $G_{1}(0)=G_{2}(0)=G_{3}(0)=0$. Let $G=\left(G_{1}, G_{2}, G_{3}\right)$. Let $B=B_{1}=\mathbb{R}^{3}, \Omega=\bar{U}(0,1), x^{*}=$ $(0,0,0)^{T}$. Define function $G$ on $\Omega$ for $v=\left(v_{1}, v_{2}, v_{3}\right)^{T}$ by

$$
G(v)=\left(e^{v_{1}}-1, \frac{e-1}{2} v_{2}^{2}+v_{2}, v_{3}\right)^{T}
$$

Then, we get

$$
G^{\prime}(v)=\left[\begin{array}{ccc}
e^{x} & 0 & 0 \\
0 & (e-1) v_{2}+1 & 0 \\
0 & 0 & 1
\end{array}\right]
$$

so $\psi_{0}(t)=(e-1) t, \psi(t)=e^{\frac{1}{e-1}} t, \omega(t)=e^{\frac{1}{e-1}}$ and $K=e$ is the Lipschitz constant on $\Omega$ and $\rho_{T}$ is given in $[29,35]$. Then, the radii:

$$
R_{1}=0.3827=\rho_{A}=\frac{2}{2(e-1)+e^{\frac{1}{e-1}}}, R_{2}=0.3061=R, \rho_{T}=\frac{2}{3 K}=0.2453
$$

EXAMPLE 5.4. Consider $B=B_{1}=C[0,1], \Omega=\bar{U}(0,1)$ and $Q: \Omega \longrightarrow B_{1}$ defined by

$$
Q(\varsigma)(x)=\varrho(x)-5 \int_{0}^{1} x \theta \varsigma(\theta)^{3} d \theta
$$

We obtain

$$
Q^{\prime}(\varsigma(\xi))(x)=\xi(x)-15 \int_{0}^{1} x \theta \varsigma(\theta)^{2} \xi(\theta) d \theta, \text { for each } \xi \in D .
$$

Then, since $x^{*}=0$, we set $\psi_{0}(t)=7.5 t, \psi(t)=15 t, \omega(t)=15$ and $K=15$. Then, the radii:

$$
R_{1}=0.0667=\rho_{A}=\frac{2}{2(7.5)+15}, R_{2}=0.0290=R, \rho_{T}=\frac{2}{3 K}=0.0444
$$

Notice that in the last two examples $\rho_{A}$ is the radius given by us in [1-7] and is the largest. 


\section{CONCLUSION}

We have provided sufficient convergence criterion for the semi-local and local convergence of two-step methods. Upon specializing the parameters involved we show that although our majorizing sequence is more general than earlier ones: Convergence criteria are weaker (i.e., the utility of the methods is extended); the upper error estimates are more accurate (i.e. at least as few iterates are required to achieve a predecided error tolerance) and we have an at least as large ball containing the solution. These benefits are obtained without additional hypotheses. According to our new technique we locate a more accurate domain than before containing the iterates resulting to more accurate (at least as small) Lipschitz condition.

Our theoretical results are further justified using numerical experiments.

\section{REFERENCES}

[1] I.K. Argyros, On the Newton - Kantorovich hypothesis for solving equations, J. Comput. Math. 169 (2004), 315-332, https://doi.org/10.1016/j.cam.2004.01.029

[2] I.K. Argyros, Computational theory of iterative methods. Series: Studies in Computational Mathematics, 15, Editors: C.K. Chui and L. Wuytack, Elsevier Publ. Co. New York, U.S.A, 2007.

[3] I.K. Argyros, Convergence and Applications of Newton-type Iterations, Springer Verlag, Berlin, Germany, (2008), https://doi.org/10.1007/978-0-387-72743-1.

[4] I.K. Argyros, S. Hilout, Weaker conditions for the convergence of Newton's method. J. Complex. 28 (2012), 364-387, https://doi.org/10.1016/j.jco.2011.12.003.

[5] I.K. Argyros, S. Hilout, On an improved convergence analysis of Newton's method, Appl. Math. Comput. 225 (2013), 372-386, https://doi.org/10.1016/j.amc.2013.09.049.

[6] I.K. Argyros, A.A. Magréñan, Iterative methods and their dynamics with applications, CRC Press, New York, USA, 2017.

[7] I.K. Argyros, A.A. Magréñan, A contemporary study of iterative methods, Elsevier (Academic Press), New York, 2018, https://www.elsevier.com/books/a-contemporary-study-of-iterative-methods/ magrenan/978-0-12-809214-9.

[8] R. Behl, P. Maroju, E. Martinez, S. Singh, A study of the local convergence of a fifth order iterative method, Indian J. Pure Appl. Math. 51 (2020), 439-455, https://doi .org/10.1007/s13226-020-0409-5.

[9] E. Cătinaş, The inexact, inexact perturbed, and quasi-Newton methods are equivalent models, Math. Comp. 74 (2005), 291-301, https://citeseerx. ist.psu. edu/viewdoc/download?doi=10.1.1.96.1713\&rep=rep1\& type $=$ pdf.

[10] X. Chen, T. Yamamoto, Convergence domains of certain iterative methods for solving nonlinear equations, Numer. Funct. Anal. Optim. 10 (1989), 37-48, https://doi.org/10.1080/01630568908816289.

[11] J.E. Dennis Jr., On Newton-like methods. Numer. Math. 11 (1968), 324-330, https://doi.org/10.1007/ BF02166685.

[12] J.E. Dennis Jr., R.B. Schnabel, Numerical methods for unconstrained optimization and nonlinear equations, SIAM, Philadelphia, 1996. First published by Prentice-Hall, Englewood Cliffs, New Jersey, (1983), https://epubs. siam.org/doi/pdf/10.1137/1.9781611971200.fm.

[13] P. Deuflhard, G. Heindl, Affine invariant convergence theorems for Newton's method and extensions to related methods. SIAM J. Numer. Anal. 16 (1979), 1-10, https://doi.org/10.1137/0716001. 
[14] P. Deuflhard, Newton methods for nonlinear problems. Affine invariance and adaptive algorithms, Springer Series in Computational Mathematics, 35, Springer - Verlag, Berlin. (2004), https://www.springer.com/gp/book/ 9783540210993.

[15] S. Erden, H. Budak, M.Z. Sarikaya, Fractional Ostrowski type inequalities for functions of bounded variaton with two variables, Miskolc Math. Notes 21 (2020), 171-188. https://doi .org/10.18514/MMN . 2020. 3076.

[16] J. A. Ezquerro, M. A. Hernandez, Newton's method: An updated approach of Kantorovich's theory, Cham Switzerland, (2018), https://WwW. springer. com/gp/book/9783319559759.

[17] M. Grau-Sánchez, À. Grau, M. Noguera, Ostrowski type methods for solving systems of nonlinear equations. Appl. Math. Comput. 281 (2011), 2377-2385, https://doi.org/10.1016/j . amc. 2011.08.011.

[18] J.M. Gutiérrez, Á.A. Magreñán, N. Romero, On the semilocal convergence of Newton-Kantorovich method under center-Lipschitz conditions, Appl. Math. Comput. 221 (2013), 79-88, https://doi.org/10.1016/j.amc.2013. 05.078.

[19] M.A. Hernandez, N. Romero, On a characterization of some Newton-like methods of $R-$ order at least three, J. Comput. Appl. Math. 183 (2005), 53-66, https://doi.org/10.1016/j . cam. 2005.01.001.

[20] L.V. Kantorovich, G.P. Akilov, Functional Analysis, Pergamon Press, Oxford, (1982).

[21] A.A. Magréñan, I.K. Argyros, J.J. Rainer, J.A. Sicilia, Ball convergence of a sixth-order Newton-like method based on means under weak conditions, J. Math. Chem. 56 (2018), 2117-2131, https://doi.org/10.1007/ s10910-018-0856-y.

[22] A.A. Magréñan, J.M. Gutiérrez, Real dynamics for damped Newton's method applied to cubic polynomials, J. Comput. Appl. Math. 275 (2015), 527-538, https://dl . acm.org/doi/abs/10.5555/2946148. 2946231.

[23] M.Z. Nashed, X. Chen, Convergence of Newton-like methods for singular operator equations using outer inverses, Numer. Math. 66 (1993), 235-257, https://doi.org/10.1007/BF01385696.

[24] L.M. Ortega, W.C. Rheinboldt" Iterative Solution of Nonlinear Equations in Several Variables, Academic press, New York, (1970), https://www.elsevier.com/books/ iterative-solution-of-nonlinear-equations-in-several-variables/ortega/978-0-12-528550-6.

[25] A. M. Ostrowski, Solution of equations in Euclidean and Banach spaces, Elsevier, 1973.

[26] F.A. Potra, V. Pták, Nondiscrete induction and iterative processes. Research Notes in Mathematics, 103. Pitman(Advanced Publishing Program), Boston, MA. (1984), http://ww. sciepub. com/reference/50811.

[27] P.D. Proinov, General local convergence theory for a class of iterative processes and its applications to Newton's method, J. Complex. 25 (2009), 38-62, https://doi.org/10.1016/j.jco.2008.05.006.

[28] M.A. Ragusa, Parabolic Herz spaces and their applications, Appl. Math. Lett. 25 (2012), 1270-1273, https: //doi.org/10.1063/1.3498444

[29] W.C. Rheinboldt, An adaptive continuation process of solving systems of nonlinear equations. Polish Academy of Science, Banach Ctr. Publ. 3 (1978), 129-142, https://eudml.org/doc/208686.

[30] S.M. Shakhno, O.P. Gnatyshyn, , On an iterative algorithm of order 1.839... for solving nonlinear least squares problems, Appl. Math. Appl. 161 (2005), 253-264, https://doi.org/10.1016/j . amc. 2003.12.025.

[31] S.M. Shakhno, R.P. lakymchuk, H.P. Yarmola, Convergence analysis of a two step method for the nonlinear squares problem with decomposition of operator, J. Numer. Appl. Math. 128 (2018), 82-95, https://hal. archives-ouvertes.fr/hal-01857847/document.

[32] J.R. Sharma, R.K. Guha, R. Sharma, An efficient fourth order weighted - Newton method for systems of nonlinear equations. Numer. Algorithms, 62 (2013), 307-323, https://doi.org/10.1007/s11075-012-9585-7.

[33] F. Soleymani, T. Lotfi, P. Bakhtiari, A multi-step class of iterative methods for nonlinear systems. Optim. Lett. 8 (2014), 1001-1015, https://doi.org/10.1007/s11590-013-0617-6. 
[34] J.F. Steffensen, Remarks on iteration. Skand Aktuar Tidsr. 16 (1993), 64-72, https://doi.org/10.1080/ 03461238.1933 .10419209$.

[35] J.F. Traub, Iterative methods for the solution of equations prentice Hall, New Jersey, U.S.A, (1964), https://doi. $\mathrm{org} / 10.1017 / \mathrm{S} 0008439500028125$.

[36] J.F. Traub, A.G. Werschulz, Complexity and information, Lezioni Lince.[Lincei Lectures] Cambridge University Press, Cambridge, 1998, xii+139 pp.

[37] J.F. Traub, Wozniakowski, H, Path integration on a quantum computer, Quant. Inf. Process. 1 (2002), 356-388, https://arxiv.org/abs/quant-ph/0109113.

[38] T. Yamamoto, A convergence theorem for Newton-like methods in Banach spaces. Numer. Math. 51 (1987), 545-557, https://eudml.org/doc/133212.

[39] R. Verma, New Trends in Fractional Programming, Nova Science Publisher, New York, USA, (2019).

[40] L. Xu, Y.-M. Chu, S. Rashid, A.A. El-Deeb, K.S. Nisar, On new unifed bounds for a family of functions via fractional q-calculus theory, J. Funct. Space 2020 (2020), 4984612, https://doi.org/10.1155/2020/4984612.

[41] P.P. Zabrejko, D.F. Nguen, The majorant method in the theory of Newton-Kantorovich approximations and the Pták error estimates, Numer. Funct. Anal. Optim. 9 (1987), 671-684, https://doi .org/10 . 1080/01630568708816254. 